\title{
A Modification of Gustavsen and Semlyen's vector fitting algorithm for overestimated model order
}

This paper was downloaded from TechRxiv (https://www.techrxiv.org).

LICENSE

CC BY 4.0

SUBMISSION DATE / POSTED DATE

$26-11-2021 / 06-12-2021$

CITATION

Mackay, Andrew (2021): A Modification of Gustavsen and Semlyen's vector fitting algorithm for overestimated model order. TechRxiv. Preprint. https://doi.org/10.36227/techrxiv.17086130.v1

DOI

10.36227/techrxiv.17086130.v1 


\title{
A Modification of Gustavsen and Semlyen's vector fitting algorithm for overestimated model order
}

\author{
A. J. Mackay
}

\begin{abstract}
Gustavsen and Semlyen's vector fitting algorithm (VFA) is popular for the determination of the poles and residues of a sampled response function, modelled as a finite order rational function. However, it does not always perform well when the original function is of infinite order and/or the model order is overestimated. This article is concerned with a novel "null-space" modification of the VFA that provides an accurate representation of the original function when the model order is large and when the original VFA performs poorly. The null-space method is parameterised by a single control parameter, $L$, which can be adjusted to improve accuracy. Comparisons are made with the original VFA and another of Gustavsen's modifications.
\end{abstract}

Index Terms-Rational Approximation, System identification, Vector Fitting (VF).

\section{INTRODUCTION}

I $\mathrm{T}$ is frequently the case that a complex valued response function, such as an input impedance $Z(\omega)$, needs to be determined as a real function of the Laplace transform variable $s=j \omega$. If $Z(\omega)$ can be evaluated at sampled real frequencies, $\omega=\omega_{i}$, and can be approximated by a finite order rational function a number of algorithms are available for this task. A popular one is Gustavsen and Semlyen's vector fitting algorithm (VFA) [1] which is computationally fast and relatively simple to implement. Subsequently, other variants of this algorithm have been developed [2], [3]. While these variants can locate the poles and zeros of finite order rational response functions to high accuracy, they can sometimes completely fail when the input function is of infinite order and the model order is taken too large. Ideally, the model order should be capable of being indefinitely increased so as to find a point where the fitting error is acceptably small.

In this article, a new modification is described which is based on the null eigenvalue variant described in [3]. In the null eigenvalue method, an error residual $E_{1}$ is small if the smallest eigenvalue magnitude is small and at each stage of an iteration process an eigenvector of residuals is estimated subject to an eigenvector normalisation of the unknown parameters. Unfortunately, the method is not particularly robust. Rather than determine a single eigenvector of residues, associated with the smallest eigenvalue magnitude, in this new "nullspace" method, all the eigenvectors are selected associated with small eigenvalue magnitudes. The linear combination of vectors is chosen that minimises a second error residual, $E_{2}$.

The method has the potential to determine a viable set of residues that minimises fitting error at each stage in the iteration cycle when there are many eigenvalues with small

A. J. Mackay is principal consultant at Universal Electromagnetics Ltd., UK. (e-mail: andrew@ueltd.co.uk). magnitudes, which occurs when the model order is chosen to be large. This frequently results in good accuracy, achieved in a small number of iterations. The algorithm is described and comparisons made with predictions using the original VFA and Gustavsen's second variant [2]. A more detailed report is available elsewhere [4] presenting further examples and considering some other variants of the method.

\section{The Vector Fitting Algorithm (VFA)}

The VFA assumes that the response function can be modelled as a finite order rational function of the form,

$$
Z(s)=\frac{P(s)}{Q(s)}=\sum_{m=1}^{N} \frac{r_{m}}{s-a_{m}}+d+s e
$$

where there are $N$ poles and the order of the polynomial $Q(s)$ is $N$. Poles exist is complex conjugate pairs or are real. If there are $P$ complex pole pairs grouped sequentially and ordered first on the $m$-list, then the complex poles are specified by complex values for $a_{m}$ and $r_{m}$ with $a_{2 m-1}=a_{2 m}^{\star}$ and $r_{2 m-1}=r_{2 m}^{\star}$ (where the superscript $\star$ represents the complex conjugate). The remaining $R=N-2 P$ poles take $a_{m}$ and $r_{m}$ to be real valued. To simplify the description of the algorithm it is assumed that the real valued coefficients $d$ and $e$ are always present and determined by the fitting process, though this need not be the case [4]. The VFA also forces all poles to have negative real part. The form (1) and the requirements on the poles are necessary but not sufficient to ensure that $Z(s)$ is a positive real function. However, no further algorithmic constraints are applied so it is possible to model non positive real functions capable of exhibiting system gain and the example presented later features such a function, defined using the poles and residues specified in example 4 of [1].

The VFA assumes an auxiliary function $\sigma(s)$ such that,

$$
\sigma(s) Z(s)=p(s)
$$

for all $N_{f}$ frequencies $\omega=\omega_{i}$ and $s=s_{i}=j \omega_{i}$, for $i=1$ to $N_{f}$ for which data is available. It is assumed that

$$
\sigma(s)=\sum_{m=1}^{N} \frac{\tilde{r}_{m}}{s-q_{m}}+\tilde{d}
$$

and

$$
p(s)=\sum_{m=1}^{N} \frac{r_{m}}{s-q_{m}}+d+s e
$$

where $q_{m}$ are estimates of the pole positions whose values, prior to iteration, may be fairly arbitrary [1]. Equation (3) 
comes from [2] for which $\tilde{d}$ is not necessarily unity. In the original VFA [1], $\tilde{d}=1$. In the second variant [2], it is defined by,

$$
\Re\left\{\sum_{k=1}^{N_{f}} w\left(\omega_{k}\right)\left(\sum_{m=1}^{N} \frac{\tilde{r}_{m}}{s-q_{m}}+\tilde{d}\right)\right\}=N_{f}
$$

extending the definition slightly by including a weight function $w(\omega)$, such that $w(\omega)$ is positive real when $\omega=\omega_{i}$ and normalised such that,

$$
\frac{1}{N_{f}} \sum_{i=1}^{N_{f}} w\left(\omega_{i}\right)=1
$$

For a given set of $q_{m}$, the left and right hand sides of (2) are linear in the unknown coefficients $\tilde{r}_{m}, r_{m}, a_{m} d$ and $e$ and (2) can be solved in a least squares sense to obtain these unknown coefficients, subject to the constraint on $\tilde{d}$. At each iteration $q_{m}$ are determined from the previously estimated values for $\tilde{r}_{m}$ and the previous values for $q_{m}$ and $\tilde{d}$, calculated using a companion matrix to determine the zeros of $\sigma(s)$ [2],[4].

The least squares solution in the above VFA variants minimises the residual error,

$$
E_{1}=\sum_{i=1}^{N_{f}}\left|\varepsilon_{i}^{(1)}\right|^{2}
$$

where

$$
\varepsilon_{i}^{(1)}=w\left(\omega_{i}\right)\left(\sigma\left(s_{i}\right) Z\left(s_{i}\right)-p\left(s_{i}\right)\right), \text { for } i \in\left[1, N_{f}\right]
$$

For diagnostic purposes the related RMS and relative error associated with $E_{1}$, may be defined by,

$$
E_{1}^{(r m s)}=\sqrt{\frac{1}{N_{f}} \sum_{i=1}^{N_{f}}\left|\varepsilon_{i}^{(1)}\right|^{2}}
$$

and,

$$
E_{1}^{(r e l)}=\frac{E_{1}^{(r m s)}}{\sqrt{\frac{1}{N_{f}} \sum_{i=1}^{N_{f}}\left|f\left(\omega_{i}\right)\right|^{2}}}
$$

where $E_{1}^{(r m s)}$ is the RMS error illustrated in examples in [2] and $f(\omega)=w(\omega) Z(s)$ is the weighted complex impedance.

In this article a second error measure,

$$
E_{2}=\sum_{i=1}^{N_{f}}\left|\varepsilon_{i}^{(2)}\right|^{2}
$$

is also required, where

$$
\varepsilon_{i}^{(2)}=w\left(\omega_{i}\right)\left(Z\left(s_{i}\right)-p\left(s_{i}\right)\right), \text { for } i \in\left[1, N_{f}\right]
$$

This is a direct measure of the error between the initial data and the model with a relative error defined by,

$$
E_{2}^{(r e l)}=\frac{E_{2}^{(r m s)}}{\sqrt{\frac{1}{N_{f}} \sum_{i=1}^{N_{f}}\left|f\left(\omega_{i}\right)\right|^{2}}}
$$

In general, at any stage in the iteration process, $E_{1}$ and $E_{2}$ can differ significantly.

If the sub-vector of terms, $\tilde{\boldsymbol{r}}$, represents the terms $\tilde{r}_{i}$ and $\boldsymbol{r}$ represents terms $r_{i}$ then the full vector of unknowns is defined by $\boldsymbol{x}=[\tilde{\boldsymbol{r}} ; \boldsymbol{r} ; \tilde{d}, d, e]^{T}$. It is assumed here that the terms in the sub-vectors are grouped as,

$\tilde{\boldsymbol{r}}=\left[\left(\tilde{r}_{1}^{\prime}, \tilde{r}_{1}^{\prime \prime}\right),\left(\tilde{r}_{2}^{\prime}, \tilde{r}_{2}^{\prime \prime}\right), \ldots,\left(\tilde{r}_{P}^{\prime}, \tilde{r}_{P}^{\prime \prime}\right) ; \tilde{r}_{2 P+1}, \tilde{r}_{2 P+2}, \ldots, \tilde{r}_{N}\right]^{T}$

$\left.\boldsymbol{r}=\left[\left(r_{1}^{\prime}, r_{1}^{\prime \prime}\right), r_{2}^{\prime}, r_{2}^{\prime \prime}\right), \ldots,\left(r_{P}^{\prime}, r_{P}^{\prime \prime}\right) ; r_{2 P+1}, r_{2 P+2}, \ldots, r_{N}\right]^{T}$

where the complex residues are expressed in terms of their real and imaginary parts, $r_{i}=r_{i}^{\prime}+j r_{i}^{\prime \prime}$ for $i=1$ to $P$, and similarly $\tilde{r}_{i}=\tilde{r}_{i}^{\prime}+j \tilde{r}_{i}^{\prime \prime}$. Let the complex rectangular matrix,

$$
A=\left(\begin{array}{l}
\boldsymbol{A}_{1} \\
\boldsymbol{A}_{2} \\
. . \\
. . \\
\boldsymbol{A}_{N_{f}}
\end{array}\right)
$$

where,

$$
\boldsymbol{A}_{i}=\left[\boldsymbol{A}_{i}^{(1)} ; \boldsymbol{A}_{i}^{(2)} ; w\left(\omega_{i}\right) Z\left(s_{i}\right) ;-w\left(\omega_{i}\right),-w\left(\omega_{i}\right) s_{i}\right]
$$

and the sub-vectors $\boldsymbol{A}_{i}^{(1)}$ and $\boldsymbol{A}_{i}^{(2)}$ are defined by,

$$
\boldsymbol{A}_{i}^{(1)}=-Z\left(s_{i}\right) \boldsymbol{A}_{i}^{(2)}
$$

and

$$
\begin{aligned}
\boldsymbol{A}_{i}^{(2)} & =-w\left(\omega_{i}\right)\left[\left(\frac{1}{s_{i}-q_{1}}+\frac{1}{s_{i}-q_{1}^{\star}}, \frac{j}{s_{i}-q_{1}}-\frac{j}{s_{i}-q_{1}^{\star}}\right),\right. \\
& \ldots\left(\frac{1}{s_{i}-q_{P}}+\frac{1}{s_{i}-q_{P}^{\star}}, \frac{j}{s_{i}-q_{P}}-\frac{j}{s_{i}-q_{P}^{\star}}\right) \\
& \left.\frac{1}{s_{i}-q_{2 P+1}}, \frac{1}{s_{i}-q_{2 P+2}}, \ldots, \frac{1}{s_{i}-q_{2 P+R}}\right]
\end{aligned}
$$

In the null eigenvalue method, $\boldsymbol{x}$ is determined by,

$$
\boldsymbol{x}=\alpha \hat{\boldsymbol{x}}_{0}
$$

where $\hat{\boldsymbol{x}}_{0}$ is the eigenvector corresponding to the smallest eigenvalue magnitude $|\lambda|_{\text {min }}$ of the eigenvalue decomposition,

$$
B \hat{\boldsymbol{x}}=\lambda \hat{\boldsymbol{x}}
$$

where the square real-valued matrix $B$ is defined by,

$$
B=\hat{A}^{T} \hat{A}=A^{\prime T} A^{\prime}+A^{\prime \prime T} A^{\prime \prime}
$$

where the $T$ represents the matrix transpose and $A^{\prime}$ and $A^{\prime \prime}$ represent the real and imaginary parts of the matrix $A$. The unknown real coefficient $\alpha$ need not be calculated in order to calculate revised estimates for $q_{i}$, since these are independent of $\alpha$, but it is necessary in order to define $E_{2}$. For this purpose it may be calculated as that value which minimises $E_{2}$. Details of this procedure and of the implementation of the original and second variant of the VFA are given in [4].

In the null-space method, central to this article, a threshold $L$ is chosen and all eigenvectors $\hat{\boldsymbol{x}}_{i}$ of (19) are determined such that the associated eigenvalue magnitudes $\left|\lambda_{i}\right|<L|\lambda|_{\min }$ or $\left|\lambda_{i}\right|<\epsilon_{m}|\lambda|_{\max }$ where $\epsilon_{m}$ is the computer precision (typically $\sim 10^{-16}$ when employing double precision arithmetic) and $|\lambda|_{\max }$ is the maximum eigenvalue magnitude. This set of vectors defines a subspace that represents an approximate nullspace of the matrix $B$. The vector $\boldsymbol{x}$ is then defined by $\boldsymbol{x}=\boldsymbol{x}_{s}^{\prime}$ where,

$$
\boldsymbol{x}_{s}^{\prime}=X_{r} \boldsymbol{\alpha}
$$


where $X_{r}$ is a rectangular matrix comprising the selected eigenvectors $\hat{\boldsymbol{x}}_{i}$ and the vector of coefficients $\boldsymbol{\alpha}$ is defined so as to minimise $E_{2}$. This is given by,

$$
\boldsymbol{\alpha}=-\left[\Re\left(C^{\dagger} C\right)\right]^{-1} \Re\left(C^{\dagger} \boldsymbol{f}\right)
$$

where the $\dagger$ represents the complex conjugate transpose, $f$ represents the vector of terms

$$
\boldsymbol{f}=\left[w\left(\omega_{1}\right) Z\left(s_{1}\right), w\left(\omega_{2}\right) Z\left(s_{2}\right), \ldots, w\left(\omega_{N_{f}}\right) Z\left(\omega_{N_{f}}\right)\right]^{T}
$$

and the matrix,

$$
C=A_{s} X_{r}
$$

where

$$
A_{s}=\left(\begin{array}{l}
\boldsymbol{A}_{1}^{(s)} \\
\boldsymbol{A}_{2}^{(s)} \\
. . \\
. . \\
\boldsymbol{A}_{N_{f}}^{(s)}
\end{array}\right)
$$

and the complex row vectors $\boldsymbol{A}_{i}^{(s)}$ are given by,

$$
\boldsymbol{A}_{i}^{(s)}=\left[\boldsymbol{A}_{i}^{(2)} ;-w\left(\omega_{i}\right),-w\left(\omega_{i}\right) s_{i}\right]
$$

The matrix inverse $\left[\Re\left(C^{\dagger} C\right)\right]^{-1}$ in (22) almost always exists since the number of eigenvectors forming the null-space is relatively small.

The threshold value $L$ has not been found to be critical when the model order $N$ is large. The default value is taken to be $L=1000$. When $N$ is smaller than the expected model order the value of $L$ becomes more significant and in this case smaller values of $L$ are required. In any use of this or the original VFA methods it is important to use the error measure $E_{2}$ and not $E_{1}$ as the measure of performance. Frequently $E_{1}$ can be small while $E_{2}$ is still quite large.

\section{EXAMPLE}

This example employs the (non positive real) 18 pole impedance test function defined in example 4 of [1]. Here, $Z(s)$ is defined by

$$
Z(s)=\sum_{i=1}^{18} \frac{r_{i}}{s-a_{i}}+d+e s
$$

where the poles and residues are defined in table I, prior to frequency scaling.

Uniform sampling is assumed with $N_{f}=200$ samples over the frequency range $f_{\min }=500 \mathrm{~Hz}$ to $f_{\max }=100 \mathrm{kHz}$ with equal weight, $w\left(\omega_{i}\right)=1$. Frequency scaling is assumed with all angular frequencies $\omega$ defined relative to the maximum angular frequency $\omega_{\max }=2 \pi f_{\max }$. Initial pole estimates $q_{i}$ are taken to be complex with imaginary parts uniformly spread over the sample range with real part $-0.001 \omega_{\max }$. The first three figures show the base-10 logarithm of the relative errors $E_{1}^{(r e l)}$ and $E_{2}^{(r e l)}$ as a function of the iteration number. Fig. 1 shows the performance of the original [1] VFA, Fig. 2 shows the performance of the second variant [2] and Fig. 3 shows the performance of the null-space method assuming $N=20$ and $N=40$. Here, the null-space method is used with the default value of $L=1000$ to define the size of the null-space. With
TABLE I: Poles and residues

\begin{tabular}{|l|l|}
\hline$a_{i}(\mathrm{~Hz})$ & $r_{i}(\mathrm{~Hz})$ \\
\hline-4500 & -3000 \\
-41000 & -83000 \\
$-100.0 \pm \mathrm{j} 5000.0$ & $-5.0 \pm \mathrm{j} 7000.0$ \\
$-120.0 \pm \mathrm{j} 15000.0$ & $-20.0 \pm \mathrm{j} 18000.0$ \\
$-3000.0 \pm \mathrm{j} 35000.0$ & $6000.0 \pm \mathrm{j} 45000.0$ \\
$-200.0 \pm \mathrm{j} 45000.0$ & $40.0 \pm \mathrm{j} 60000.0$ \\
$-1500.0 \pm \mathrm{j} 45000.0$ & $90.0 \pm \mathrm{j} 10000.0$ \\
$-500.0 \pm \mathrm{j} 70000.0$ & $50000.0 \pm \mathrm{j} 80000.0$ \\
$-1000.0 \pm \mathrm{j} 73000.0$ & $1000.0 \pm \mathrm{j} 45000.0$ \\
$-2000.0 \pm \mathrm{j} 90000.0$ & $-5000.0 \pm \mathrm{j} 92000.0$ \\
\hline$d$ (dimensionless) & $e\left(\mathrm{~Hz}^{-1}\right)$ \\
\hline 0.2 & $2.0 \times 10^{-5}$ \\
\hline
\end{tabular}

this value, 3 eigenvectors are selected when $N=20$ and 23 when $N=40$. Fig. 4 shows the magnitude of the impedance function as a function of frequency, reconstructed using the null-space method when $N=40$ after 5 iterations.

In all cases, $E_{1}^{(r e l)}$ is quite small but this is not a good measure of accuracy. When $N=20$, the original VFA converges to a result with highest accuracy, the second variant performs poorly and the null-space method performs quite well. When $N=40$ the null-space method provides a result with similar accuracy after only a few iterations while the other two methods perform poorly.

\section{REFERENCES}

[1] B. Gustavsen, A. Semlyen, "Rational approximation of frequency domain responses by vector fitting," in IEEE Trans. Power Delivery, Vol. 14, No. 3, pp 1052-1061, July 1999.

[2] B. Gustavsen, "Improving the pole relocating properties of vector fitting," in IEEE Trans. Power Delivery, Vol. 21, No. 3, pp 1587-1592, July 2006.

[3] D. Deschrijver, B. Gustavsen, T. Dhaene, "Advancements in iterative methods for rational approximation in the frequency domain", in IEEE Trans. Power Delivery, Vol. 22, No. 3, pp 1633-1642, July 2007.

[4] A. J. Mackay, "Variants of Gustavsen and Semlyen's vector fitting algorithm for impedance function characterisation", UELtd, Leominster, Herefordshire, UK, Tech. Rep. UELtd/AJM/PV/Zed_estimation/v1.0, 2021, Available: www.ueltd.co.uk 


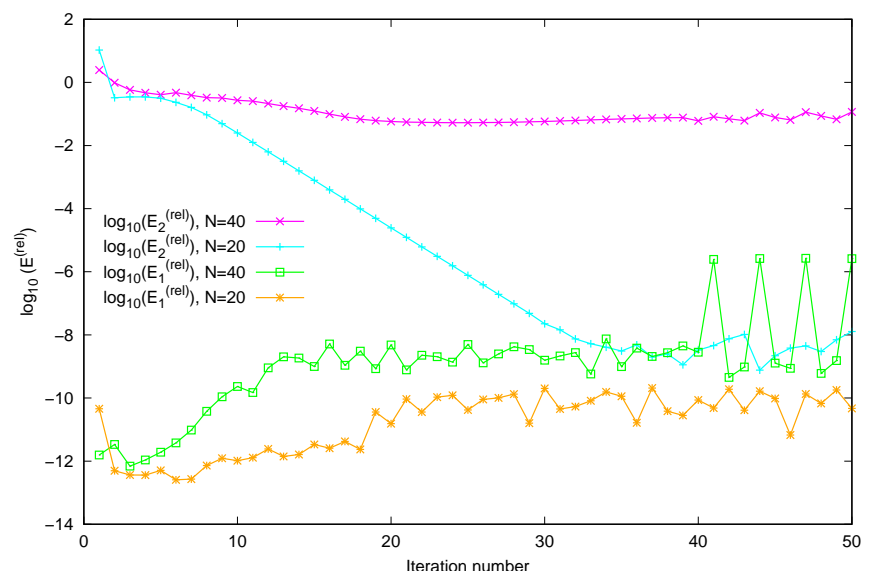

Fig. 1: Original VFA. Logarithm of $E_{1}^{(r e l)}$ and $E_{2}^{(r e l)}$ plotted as a function of iteration number.

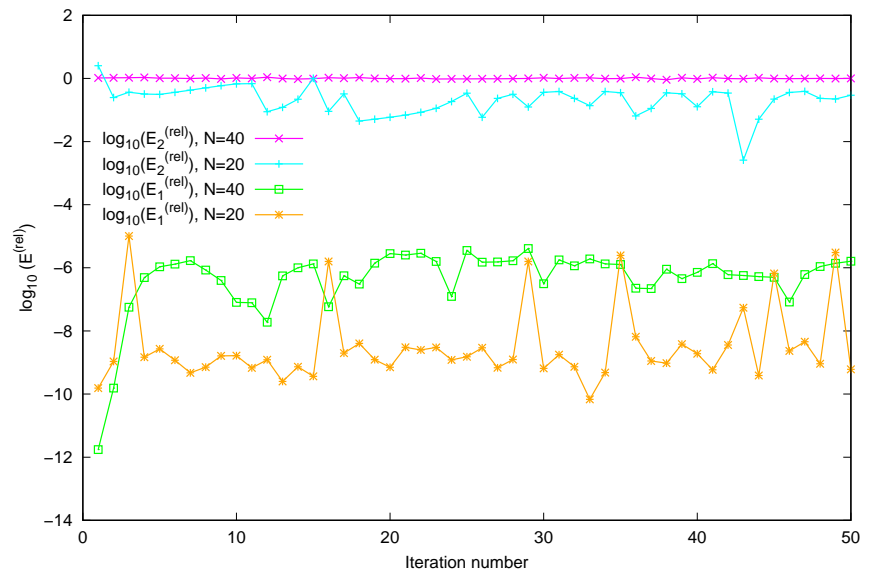

Fig. 2: Second variant VFA. Logarithm of $E_{1}^{(r e l)}$ and $E_{2}^{(r e l)}$ plotted as a function of iteration number.

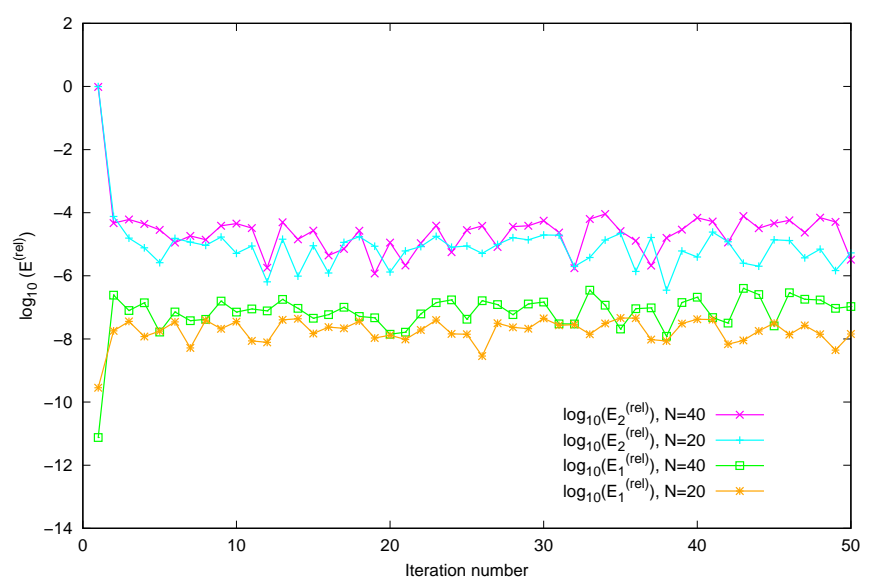

Fig. 3: Null-space method. Logarithm of $E_{1}^{(r e l)}$ and $E_{2}^{(r e l)}$ plotted as a function of iteration number.

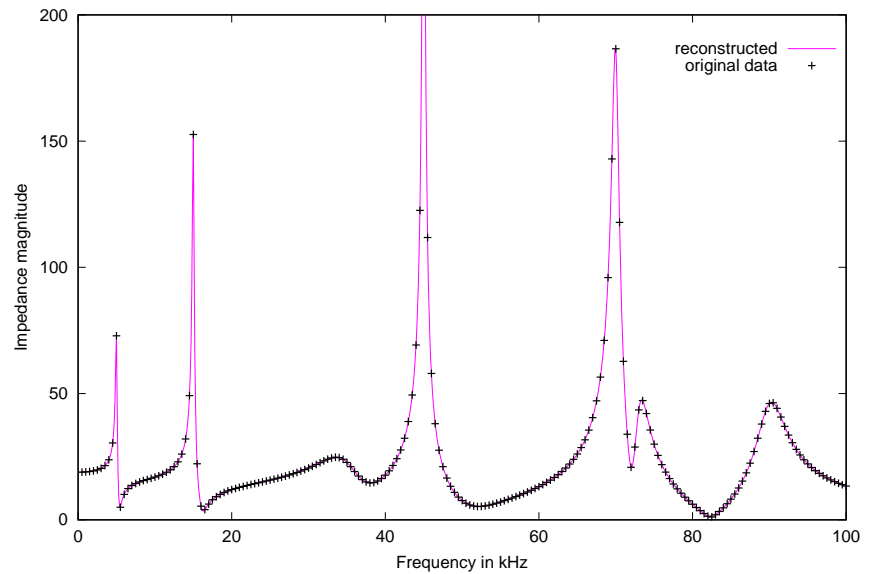

Fig. 4: Null-space method. Impedance magnitude reconstruction as a function of frequency for the case $N=40$. 